\title{
Recent results from NA62
}

\author{
Stoyan Trilov*t \\ University of Bristol \\ E-mail: stoyan.trilovecern.ch
}

\begin{abstract}
$K^{+} \rightarrow \pi^{+} v \bar{v}$ is one of the theoretically cleanest meson decay where to look for indirect effects of new physics complementary to LHC searches. The NA62 experiment at CERN SPS is designed to measure the branching ratio of the $K^{+} \rightarrow \pi^{+} v \bar{v}$ decay with $10 \%$ precision. NA62 took data in 2015 and 2016 reaching the Standard Model sensitivity. The experiment will be reviewed, and recent results and prospects will be presented, also on heavy neutrinos limits.
\end{abstract}

XVII International Conference on Hadron Spectroscopy and Structure - Hadron2017

25-29 September, 2017

University of Salamanca, Salamanca, Spain

*Speaker.

${ }^{\dagger}$ On behalf of the NA62 Collaboration: R. Aliberti, F. Ambrosino, R. Ammendola, B. Angelucci, A. Antonelli, G. Anzivino, R. Arcidiacono, M. Barbanera, A. Biagioni, L. Bician, C. Biino, A. Bizzeti, T. Blazek, B. Bloch-Devaux, V. Bonaiuto, M. Boretto, M. Bragadireanu, D. Britton, F. Brizioli, M.B. Brunetti, D. Bryman, F. Bucci, T. Capussela, A. Ceccucci, P. Cenci, V. Cerny, C. Cerri, B. Checcucci, A. Conovaloff, P. Cooper, E. Cortina Gil, M. Corvino, F. Costantini, A. Cotta Ramusino, D. Coward, G. D’Agostini, J. Dainton, P. Dalpiaz, H. Danielsson, N. De Simone, D. Di Filippo, L. Di Lella, N. Doble, B. Dobrich, F. Duval, V. Duk, J. Engelfried, T. Enik, N. Estrada-Tristan, V. Falaleev, R. Fantechi, V. Fascianelli, L. Federici, S. Fedotov, A. Filippi, M. Fiorini, J. Fry, J. Fu, A. Fucci, L. Fulton, E. Gamberini, L. Gatignon, G. Georgiev, S. Ghinescu, A. Gianoli, M. Giorgi, S. Giudici, F. Gonnella, E. Goudzovski, C. Graham, R. Guida, E. Gushchin, F. Hahn, H. Heath, T. Husek, O. Hutanu, D. Hutchcroft, L. Iacobuzio, E. Iacopini, E. Imbergamo, B. Jenninger, K. Kampf, V. Kekelidze, S. Kholodenko, G. Khoriauli, A. Khotyantsev, A. Kleimenova, A. Korotkova, M. Koval, V. Kozhuharov, Z. Kucerova, Y. Kudenko, J. Kunze, V. Kurochka, V.Kurshetsov, G. Lanfranchi, G. Lamanna, G. Latino, P. Laycock, C. Lazzeroni, M. Lenti, G. Lehmann Miotto, E. Leonardi, P. Lichard, L. Litov, R. Lollini, D. Lomidze, A. Lonardo, P. Lubrano, M. Lupi, N. Lurkin, D. Madigozhin, I. Mannelli, G. Mannocchi, A. Mapelli, F. Marchetto, R. Marchevski, S. Martellotti, P. Massarotti, K. Massri, E. Maurice, M. Medvedeva, A. Mefodev, E. Menichetti, E. Migliore, E. Minucci, M. Mirra, M. Misheva, N. Molokanova, M. Moulson, S. Movchan, M. Napolitano, I. Neri, F. Newson, A. Norton, M. Noy, T. Numao, V. Obraztsov, A. Ostankov, S. Padolski, R. Page, V. Palladino, C. Parkinson, E. Pedreschi, M. Pepe, M. Perrin-Terrin, L. Peruzzo, P. Petrov, F. Petrucci, R. Piandani, M. Piccini, J. Pinzino, I. Polenkevich, L. Pontisso, Yu. Potrebenikov, D. Protopopescu, M. Raggi, A. Romano, P. Rubin, G. Ruggiero, V. Ryjov, A. Salamon, C. Santoni, G. Saracino, F. Sargeni, V. Semenov, A. Sergi, A. Shaikhiev, S. Shkarovskiy, D. Soldi, V. Sougonyaev, M. Sozzi, T. Spadaro, F. Spinella, A. Sturgess, J. Swallow, S. Trilov, P. Valente, B. Velghe, S. Venditti, P. Vicini, R. Volpe, M. Vormstein, H. Wahl, R. Wanke, B. Wrona, O. Yushchenko, M. Zamkovsky, A. Zinchenko. 


\section{Heavy Neutrinos}

According to the standard model (SM) neutrinos are massless, however recent experimental results strongly indicate that neutrinos do have mass. This requires the extension of the SM to accommodate massive neutrinos. One possible extension is the neutrino minimal standard model or $(v$ MSM $)$ [1], where three right-handed neutrinos are added to the SM, with one of them having a mass of the order of $\mathrm{GeV}$.

If the neutrino mass $\left(m_{N}\right)$ satisfies, $m_{N}<m_{K}-m_{l}$, where $l$ is either an electron or a muon, then these right-handed neutrinos can be produced via the process:

$$
\Gamma\left(K^{ \pm} \rightarrow l^{ \pm} N\right)=\Gamma\left(K^{ \pm} \rightarrow l^{ \pm} v_{l}\right) \cdot \rho_{m_{N}} \cdot\left|U_{l 4}\right|^{2},
$$

in which $U_{l 4}$ is the mixing matrix, and $\rho_{m_{N}}$ is a factor accounting for the kinematic phase space and helicity suppression. Searches for such particles have been performed at NA62 using data collected in 2007 and 2015.

\subsection{NA62-2007}

In 2007 the NA62 collaboration collected approximately $6 \times 10^{7}$ kaon decays $\left(N_{K}\right)$, using the detector [2] of the previous experiment, NA48/2. The analysis [3] of this data searched for heavy neutrinos produced in the muon mode of process 1.1, known as $K \mu 2$. The analysis strategy consists of looking for a peak in the missing mass distribution, $m_{m i s s}=\sqrt{\left(P_{K^{+}}-P_{\mu^{+}}\right)^{2}}$, corresponding to the mass of the heavy neutrino. The mass range considered is $300-370 \mathrm{MeV} / \mathrm{c}^{2}$. There is no acceptance above $370 \mathrm{MeV} / \mathrm{c}^{2}$, and there are already strong upper limits for $m_{N}<300 \mathrm{MeV} / \mathrm{c}^{2}$.

If $\left|U_{l 4}\right|^{2}<10^{-4}$, the mean free path of the heavy neutrino would be approximately $10 \mathrm{~km}$ for the above mass range. This means that heavy neutrino decays inside the detector or decay volume can be neglected, since the probability is below $1 \%$. Signal acceptance was evaluated using a Monte Carlo (MC) simulation, whereas background estimation used MC and a data-driven approach.

\subsubsection{Detector}

The beam in 2007 was $6 \%$ kaons with central momentum of $74 \pm 0.1 \mathrm{GeV} / \mathrm{c}$. The fiducial volume was a $114 \mathrm{~m}$ long evacuated cylindrical tank. The momentum of daughter particles was measured by a magnetic spectrometer, composed of four drift chambers. A scintillator hodoscope (HOD) was used for triggering. An electromagnetic calorimeter filled with liquid krypton (LKr), followed the hodoscope. A muon veto system (MUV) was present, which consisted of three planes of plastic scintillator each preceded by $0.8 \mathrm{~m}$ thick iron absorber.

\subsubsection{Selection}

$K \mu 2$ decays were selected by requiring exactly one positively charged track within the geometrical acceptances of the spectrometer, HOD, LKr, and MUV. Each track also had to have geometrically associated clusters in the LKr and MUV. Events with LKr clusters with energy above $2 \mathrm{GeV}$ not associated with the track were rejected. Cuts on the decay vertex, track momentum, closest distance of approach (CDA) between the track and the beam axis were also applied. 


\subsubsection{Results}

The analysis was performed by searching for a signal in mass windows in steps of $1 \mathrm{MeV}$. Each window had a width of $\pm \sigma_{m}$, the missing mass resolution. In the absence of signal above $3 \sigma$ statistical significance, the Rolke-Lopez method [4] was used to estimate upper limits at $90 \%$ confidence level (CL) on the number of signal events $n_{U L}$, figure 1 . These limits can then be translated to limits on $B R\left(K^{+} \rightarrow \mu^{+} N\right)$ and $\left|U_{\mu 4}\right|^{2}$ using

$$
B_{U L}\left(K^{+} \rightarrow \mu^{+} N\right)=\frac{n_{U L}}{N_{K} \times A\left(m_{N}\right)}, \quad\left|U_{\mu 4}\right|_{U L}^{2}=\frac{B_{U L}\left(K^{+} \rightarrow \mu^{+} N\right)}{B_{U L}\left(K^{+} \rightarrow \mu^{+} v_{\mu}\right)} \times \frac{1}{\rho_{m_{N}}},
$$

where $A\left(m_{N}\right)$ is the signal acceptance, a function of the heavy neutrino mass, $m_{N}$.
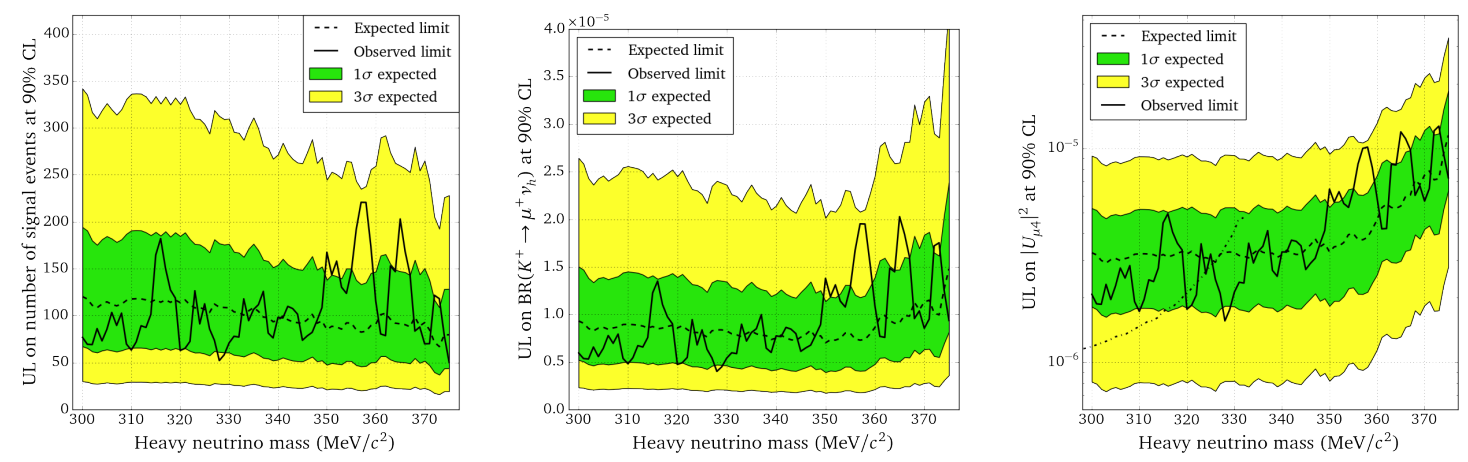

Figure 1: Expected and observed upper limits (at 90\% CL) on (from left to right) the number of $K \mu 2$ signal events $n_{U L}, B R\left(K^{+} \rightarrow \mu^{+} N\right)$, and $\left|U_{\mu 4}\right|^{2}$ at each tested mass.

\subsection{NA62-2015}

A similar analysis was performed with data taken in 2015 with the upgraded NA62 detector [5], looking for a peak in the missing mass spectrum. This search looked in the electron channel of $1.1(\mathrm{Ke} 2)$. The dataset consisted of $(3.011 \pm 0.11) \times 10^{11}$ kaon decays. The mass range used is $170-448 \mathrm{MeV} / \mathrm{c}^{2}$, since the acceptance drops off sharply above $448 \mathrm{MeV} / \mathrm{c}^{2}$. As before, heavy neutrino decays inside the detector or fiducial volume are negligible. Both MC and data-driven techniques were used to evaluate background contributions.

\subsubsection{Detector}

The beam momentum is $75 \mathrm{GeV} / \mathrm{c}, 6 \%$ kaon. The subdetectors can be placed in two categories, upstream or downstream of the fiducial volume. The upstream detectors are KTAG, GTK, and CHANTI. The KTAG is a Cerenkov detector used to identify kaons with sub 100ps time resolution. The GTK consists of three silicon pixel stations, and is used to measure the momentum of the incoming kaons. The CHANTI follows the GTK, and is designed to guard against inelastic interactions in the last GTK station.

The fiducial volume is evacuated to a pressure of $10^{-6}$ mbar. A magnetic spectrometer consisting of four STRAW tube chambers measures the momentum of the decay products. Two plastic 
scintillators, NA48-CHOD and CHOD, are involved in the triggering process. Particle identification (PID) is performed using a RICH detector, and three sets of muon veto systems, MUV1/2/3, made from a combination of plastic scintillators and iron blocks.

The IRC and SAC are used to detect small angle photons (0-1 mrad), whereas the $\mathrm{LKr}$ calorimeter handles photons with medium angles (1-8.5 mrad). The large angle veto (LAV) handles photons with angles up to $50 \mathrm{mrad}$. It is made of 12 lead glass scintillator stations placed throughout the decay region. The trigger system has three different stages, reducing the incoming rate from $5 \mathrm{MHz}$ down to a few $\mathrm{kHz}$.

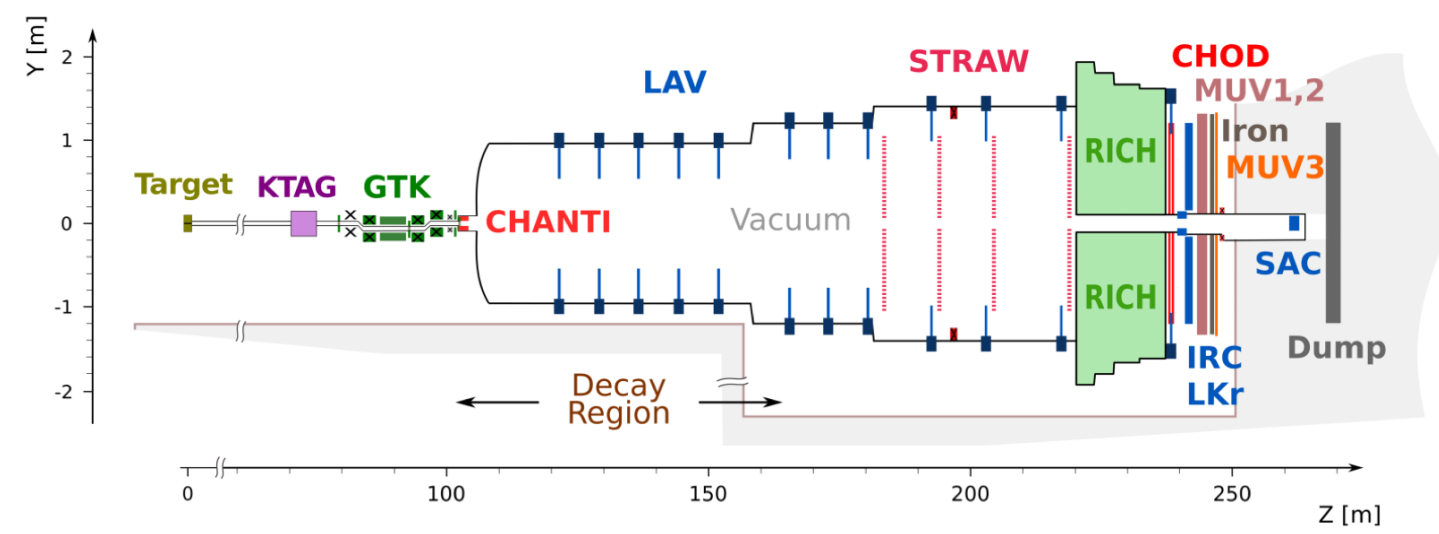

Figure 2: A schematic of the current NA62 detector.

\subsubsection{Selection}

The $K e 2$ selection requires an identified kaon in time with exactly one positively charged track in the geometrical acceptance of the spectrometer, RICH, LKr, and MUV1/2/3. PID is performed using the RICH, $\mathrm{LKr}$, and muon veto systems. Cuts on the CDA and the decay vertex are applied, as well as a photon veto.

\subsubsection{Results}

The search was performed in mass steps of $1 \mathrm{MeV} / \mathrm{c}^{2}$, where the search window was $\pm 1.5 \sigma_{m}$. No signal above $3 \sigma$ significance was observed. Upper limits at $90 \%$ CL were set on the number of signal events, and consequently on $B R\left(K^{+} \rightarrow e^{+} N\right)$ and $\left|U_{e 4}\right|^{2}$, using the Rolke-Lopez method. As can be seen from figure 3 , the new results from the two analyses either improve on or extend previous limits.

\section{2. $K^{+} \rightarrow \pi^{+} v \bar{v}$}

In the standard model, $K^{+} \rightarrow \pi^{+} v \bar{v}$ is a FCNC process, and hence it must be proceed via box or loop diagrams. It is highly suppressed with expected branching fraction of $(9.11 \pm 0.72) \times$ $10^{-11}$ [6]. The best current experimental measurement is $\left(17.3_{-10.5}^{+11.5}\right) \times 10^{-11}$ [7]. NA62 aims to significantly improve on this measurement, and reach the precision of the theoretical calculation. This is complementary to LHC physics, and would constrain several beyond the SM models [8, 9, 10]. 

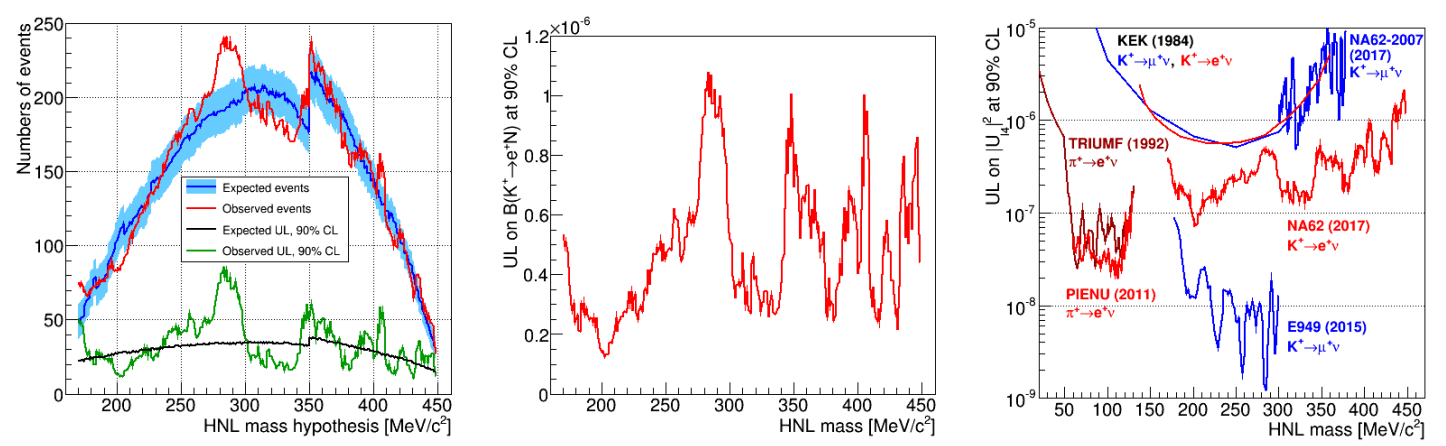

Figure 3: Expected and observed upper limits (at 90\% CL) on (from left to right) the number of $K e 2$ signal events and $B R\left(K^{+} \rightarrow e^{+} N\right)$ for each tested mass. The plot on the far right shows $\left|U_{l 4}\right|^{2}$ limits from this and other measurements.

\subsection{Analysis Strategy}

The measurement is based around four guiding principles. Precise timing: the KTAG, GTK, and RICH have a time resolution of around 100ps or better. Kinematic rejection: missing mass defined as, $m_{\text {miss }}^{2}=\sqrt{\left(P_{K^{+}}-P_{\pi^{+}}\right)^{2}}$, is used to suppress background coming from the main kaon decays at the level of $10^{4}$. Two signal regions have been defined in the $m_{\text {mass }}^{2}$ spectrum, figure 4 . Photon rejection: suppress photons coming from $\pi^{0}$ decays by an order of $10^{7}$. Separation of $\pi / \mu$ : the RICH and the calorimeters provide independent PID, leading to $10^{7} \mu$ suppression.
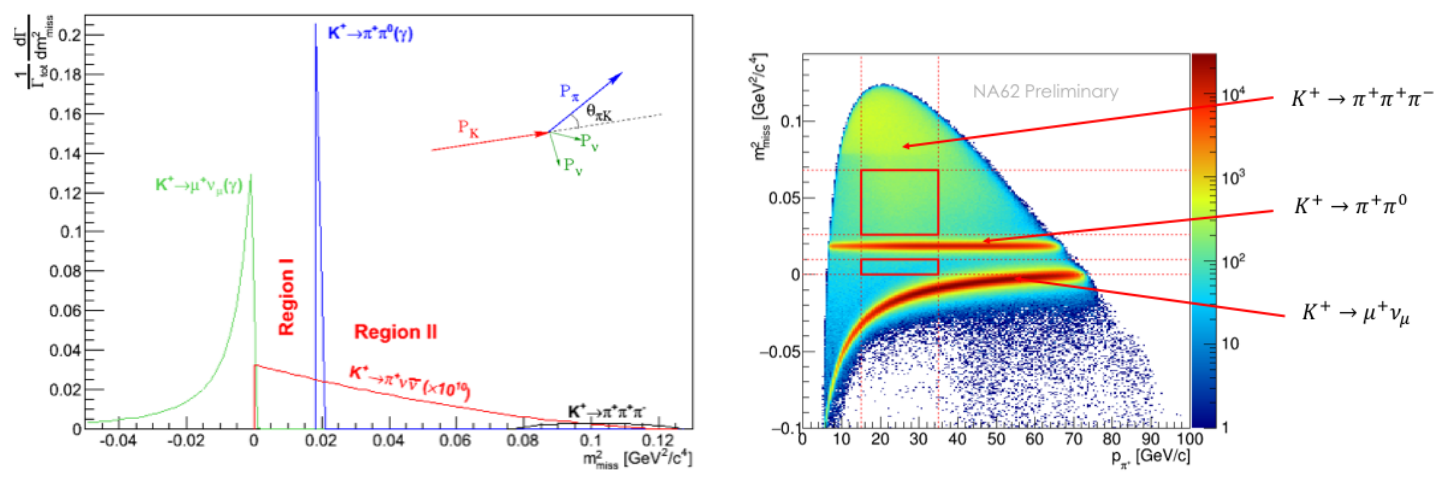

Figure 4: Left: theoretical calculation of the missing mass spectrum with signal regions 1 and 2 labelled. Right: $m_{m i s s}^{2}$ as a function of $P_{\pi^{+}}$, signal regions are enclosed in the red boxes.

\subsection{Selection}

An upstream and a downstream track are defined, i.e. a $K^{+}$and a $\pi^{+}$, and are then matched in space and time. Timing information comes from the KTAG, GTK, and RICH. At the moment, the efficiency of this procedure is $75 \%$ with $1.7 \%$ mis-tagging probability.

Next the decay signal region is defined as a function of $z_{\text {vertex }}, P_{\pi^{+}}$, and $m_{m i s s}^{2}$. Kinematic rejection is performed using three different definitions of the $m_{m i s s}^{2}$. These are $m_{m i s s}^{2}(S T R A W)=$ $\left(P_{K^{+}}^{G T K}-P_{\pi^{+}}^{S T R A W}\right)^{2}, m_{\text {miss }}^{2}(R I C H)=\left(P_{K^{+}}^{G T K}-P_{\pi^{+}}^{R I C H}\right)^{2}$ under $\pi$ hypothesis, $m_{\text {miss }}^{2}($ Beam $)=\left(P_{K^{+}}^{\text {Beam }}-\right.$ $\left.P_{\pi^{+}}^{S T R A W}\right)^{2}$ where the beam momentum has been measured using $K^{+} \rightarrow \pi^{+} \pi^{-} \pi^{+}$decays. 
Using these definitions together helps to control the non-Gaussian tails of the $m_{\text {miss }}^{2}$ distribution. The signal regions in figure 4 help suppress events coming from the main kaon decays, $B R\left(K^{+} \rightarrow \mu^{+} v\right)=63.56 \%, B R\left(K^{+} \rightarrow \pi^{+} \pi^{0}\right)=20.66 \%, B R\left(K^{+} \rightarrow \pi^{+} \pi^{-} \pi^{+}\right)=5.58 \%$. Contamination of the signal region by $K^{+} \rightarrow \pi^{+} \pi^{0}$ is of the level $6 \times 10^{-4}$, and $3 \times 10^{-4}$ for $K^{+} \rightarrow \mu^{+} v$.

The calorimetric $\pi / \mu$ separation uses a multi-variate technique, and is combined with PID from the RICH. The $\mu$ efficiencies for the calorimeters and the RICH are $10^{-5}$ and $10^{-2}$ respectively; the $\pi$ efficiency is $80 \%$ for both. A $\pi^{0}$ suppression of $(1.2 \pm 0.2) \times 10^{-7}$ was measured on a $K^{+} \rightarrow \pi^{+} \pi^{0}$ data sample.

\subsection{Results}

The full $\pi v \bar{v}$ selection was applied to $5 \%$ of 2016 data, amounting to $2.3 \times 10^{10}$ kaon decays. No signal events were observed against an expectation of 0.064 , and background expectation of 0.052 . Work is currently on-going to optimise the analysis.

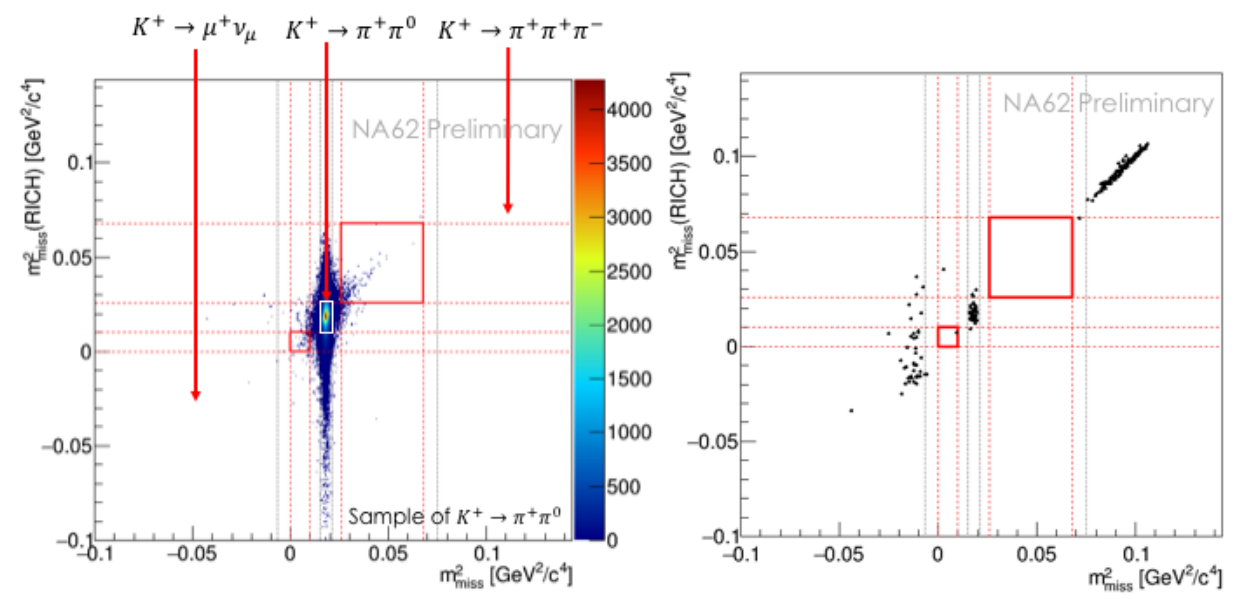

Figure 5: Left: Distribution of $m_{\text {miss }}^{2}(R I C H)$ against $m_{\text {miss }}^{2}(S T R A W)$ for a $K^{+} \rightarrow \pi^{+} \pi^{0}$ sample. Right: Same distribution after full selection has been applied, apart from the $m_{m i s s}^{2}($ Beam $)$ requirement. The event in region 1 falls outside of the $m_{\text {miss }}^{2}$ (Beam) signal region.

\section{References}

[1] T. Asaka, M. Shaposhnikov, Phys. Lett. B 620, 17 (2005)

[2] V. Fanti et al., [NA48 Collaboration], Nucl. Instrum. Meth. A 574, 433 (2007)

[3] The NA62 Collaboration, Phys. Lett. B 772, 712-718 (2017)

[4] W. A. Rolke, A. M. Lopez, Nucl. Instrum. Methods A 458, 754 (2001)

[5] The NA62 Collaboration, JINST 12, P05025 (2017)

[6] A. J. Buras, D. Buttazzo, J. Girrbach-Noe, et al., JHEP 1511 (2015)

[7] A. V. Artamonov et al. (E949 Collaboration), Phys. Rev. D 79, 092004 (2009)

[8] M. Blanke, A.J. Buras, S. Recksiegel, JHEP 0903, 108 (2009)

[9] M. Blanke, A. J. Buras, S. Recksiegel, Eur. Phys. J. C 76.no.4, 182 (2016)

[10] A. J. Buras, D. Buttazzo, R. Knegiens, JHEP 1511, 166 (2015) 